
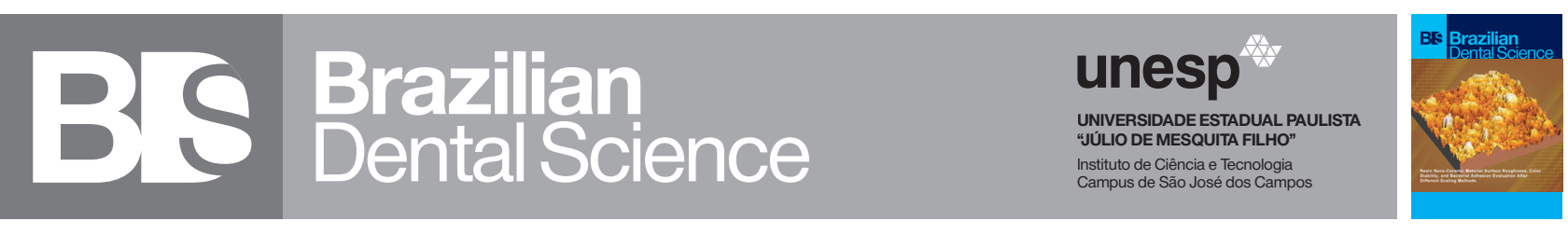

\title{
Awareness and precaution attitude of dentists as regards to risks associated with exposure to COVID-19
}

\author{
Conscientização e atitude de precaução dos dentistas em relação aos riscos associados à exposição a COVID-19
}

\author{
Merve BENLI ${ }^{1,2}$, Olivier HUCK ${ }^{3,4}$, Mutlu ÖZCAN ${ }^{5}$ \\ 1 - University of Pittsburgh, School of Dental Medicine, Department of Oral Biology, Pittsburgh, USA. \\ 2 - Istanbul University, Faculty of Dentistry, Department of Prosthodontics, Istanbul, Turkey. \\ 3 - Department of Periodontology, Faculty of Dentistry, University of Strasbourg, Strasbourg, France. \\ 4 - INSERM, UMR 1260 'Osteoarticular and Dental Regenerative Nanomedicine', Faculty of Medicine, Strasbourg, France. \\ 5 - Dental Materials Unit, Center for Dental and Oral Medicine Clinic for Fixed and Removable Prosthodontics and Dental Materials Science, \\ University of Zürich, Zürich, Switzerland.
}

\section{ABSTRACT}

Objective: Dentists need a high level of awareness to limit the spread of COVID-19 (Coronavirus disease 2019). This study aimed to evaluate the level of awareness and attitude regarding the risk associated with dental procedures among dentists. Material and Methods: An online questionnaire was submitted to dentists between April- May 2020. The questionnaire form included questions related to demographic data, the transmission characterization of SARS-CoV-2, and treatment of COVID-19 patients. Data were analyzed using IBM SPSS V23 and chi-square test ( $\mathrm{p} \leq$ 0.05). Results: A total of 3825 participants (29.1 \pm 7.6 years) were included. In COVID-19, the riskiest dental branch in terms of the risk of contamination through saliva was considered to be Periodontics (32.2\%), while the least risky branch was Orthodontics (0.2\%). Specific dental treatment procedures considered at high risk of contamination were tooth preparation (69.4\%), scaling and root planing (63.5\%), filling (53.4\%), and pulpectomy $(40.5 \%)$. The parameters of the study that differed according to gender and professional status were the viability of the virus, the risk assessment, saliva contamination risk, and aerosol-generating activities for COVID-19 ( $\mathrm{p}<0.05)$. Conclusion: Dentists were aware of the risk assessment and extra precautionary methods. However, they had limited knowledge about the viability of the virus. Dentists should be aware of recommended approaches and update their knowledge about COVID-19 to limit the spread of the disease. Since dentistry is an area

\section{RESUMO}

Objetivo: Os dentistas precisam de um alto nível de conhecimento para limitar a disseminação de COVID-19 (doença por coronavírus 2019). Este estudo teve como objetivo avaliar o nível de consciência e atitude em relação ao risco associado aos procedimentos odontológicos entre os dentistas. Material e Métodos: Um questionário online foi submetido aos dentistas entre abril e maio de 2020. O formulário do questionário incluía questões relacionadas aos dados demográficos, à caracterização da transmissão da SARS-CoV-2 e ao tratamento de pacientes com COVID-19. Os dados foram analisados usando IBM SPSS V23 e teste do qui-quadrado ( $\mathrm{p} \leq 0,05$ ). Resultados: Um total de 3825 participantes (29,1 \pm 7,6 anos) foram incluídos. Na COVID-19, o ramo odontológico de maior risco em termos de risco de contaminação pela saliva foi considerado a Periodontia (32,2\%), enquanto o ramo de menor risco foi a Ortodontia $(0,2 \%)$. Os procedimentos odontológicos específicos considerados de alto risco de contaminação foram preparo dentário $(69,4 \%)$, raspagem e alisamento radicular (63,5\%), obturação $(53,4 \%)$ e pulpectomia $(40,5 \%)$. Os parâmetros do estudo que diferiram de acordo com sexo e status profissional foram a viabilidade do vírus, a avaliação de risco, risco de contaminação da saliva e atividades geradoras de aerossol para COVID-19 ( $p<0,05$ ). Conclusão: Os dentistas estão cientes da avaliação de risco e dos métodos de precaução extra. No entanto, eles tinham conhecimento limitado sobre a viabilidade do vírus. Os dentistas devem estar cientes das abordagens recomendadas e atualizar seus conhecimentos sobre COVID-19 para limitar a propagação da doença. Como a odontologia é uma área propícia para a transmissão da COVID-19, se dentistas tiverem informações sobre 
suitable for the transmission of the COVID-19, the fact that dentists have information about the viability of this virus will be lifesaving in clinical applications.

\section{KEYWORDS}

COVID-19; SARS-CoV-2; Dentistry; Risk; Viability. a viabilidade desse vírus poderão salvar vidas em aplicações clínicas.

\section{PALAVRAS-CHAVE}

COVID-19; SARS-CoV-2; Odontologia; Risco; Viabilidade.

\section{INTRODUCTION}

$\Pi$ he coronavirus disease (COVID-19) 1 associated with SARS-CoV-2 is a novel viral infection that rapidly spreads by causing an outbreak of pneumonia in the world and has been declared as a 'pandemic disease' [1,2]. Globally, there have been nearly 90 million confirmed cases of COVID-19 and more than 2 million related deaths reported in January 2021 [3]. Since this outbreak is so devastating and fast, numerous countries have shut down schools, events, airports, sports activities, social gatherings to control the spread of the disease. Besides, some countries implemented curfews and individuals played their part in society by going into self-quarantine to limit spreading [2-4].

Being a member of the coronaviruses' family, SARS-CoV-2 is a novel RNA beta coronavirus [5]. The most associated symptoms of COVID-19 are fatigue, fever, dyspnea, and dry cough. More than $80 \%$ of patients present mild clinical symptoms and heal without specific or invasive treatment. However, about 15\% of patients are classified as severe cases, and the remaining 5\% as critical cases. In critical and severe cases, acute respiratory disease can cause kidney failure, pneumonia, and even death [6]. The two main suggested routes of SARS-CoV-2 transmission comprised the direct transmission (through sneezing, coughing, and inhalation of airborne droplets) and contact transmission (through contact with a contaminated surface or infected oral, nasal, and ocular mucosa) $[7,8]$. This virus can be viable in aerosols and survive up to 3 days on inert surfaces at room temperature increasing the risk of contamination [9]. Nevertheless, SARS-CoV-2 has been isolated from the saliva of patients diagnosed with COVID-19 and was detected up to 11 days after hospitalization [10].

The fast spread of the disease has also affected the medical community as many healthcare professionals were infected while treating symptomatic or asymptomatic patients [8]. In Turkey, the health minister announced that more than 120000 healthcare professionals have been infected with COVID-19 during this pandemic [11]. Centers for Disease Control (CDC) also reported that more than 100000 health care professionals were confirmed to have been infected during February 12-July 16, 2020 [12]. According to current data, healthcare professionals who work intraorally have been reported to be at daily risk of exposure to this virus more than other specialists [2]. Indeed, the asymptomatic incubation period is estimated to be between 2 and 12 days, while some studies have reported that the incubation period is up to 24 days [13]. Hence, without any symptoms, COVID-19 patients can pose a major threat to dentists and other members of the dental staff during treatment [7]. Nevertheless, the risk of cross-contamination is also important between patients due to the nature of routine dental treatment modalities including the use of airwater syringes, ultrasonic scalers, and air turbine handpieces, causing the emergence of significant amounts of aerosols and droplets that bounce from the oral cavity and settle on inert surfaces $[6,8]$. In a recent study that analyzed aerosol and viability of SARS-CoV-2 on inert surfaces, the viability of the virus was demonstrated as follows: up to 72 hours on plastic, 48 hours on stainless steel, 24 hours on cardboard, and 4 hours on copper. Additionally, SARS-CoV-2 was shown to remain viable in aerosol for 3 hours 
[9]. To limit the transmission of COVID-19, several guidelines and recommendations have been proposed so far by several professional organizations such as the American Dental Association (ADA), CDC, FDI World Dental Federation (FDI), Public Health England (PHE) [14-17]. Detailed plans for recommendations and clinical actions have been reported including setting up dental spaces, before/during/after dental treatment, and disinfection [18]. It has been noted that, besides standard precaution methods in general dental practice such as gloves, masks, protective eyewear, and hand hygiene procedures, use of additional personal protective equipment, disinfected environmental surfaces for dental treatments and transmission-based precautions should be implemented in dental clinics $[18,19]$. Second-level precautions consist of the use of particulate respirators (e.g., N-95 masks or FFP2/3-standard masks), high-volume evacuation (HVE) or systems, and ventilation procedures [20]. Additionally, dentists have been recommended to postpone elective dental treatment for the next weeks and to focus only on urgent treatments $[12,21,22]$. However, there is still a high demand for dental emergency care even if a decrease was observed such as in China where a 38\% decrease of dental emergencies treatment was measured during the pandemic [23]. This data indicates that the need for emergency care even during this critical time will always be imperative. Nevertheless, as far there is no clear indication on the duration of COVID-19 pandemic and associated social restrictions, the increased risk of suffering of the patients in need of urgent dental treatment implies the restart of dental care [6].

Besides acquiring and transmitting the infection between individuals or staff, the dental clinic can be a riskier environment for the spread of the virus due to the need for close contact with patients and the nature of dental treatment [24]. Although it is not considered appropriate for patients diagnosed with COVID-19 to receive dental treatment, dental emergencies may occur, and close contact cannot be avoided [8]. Dentists should therefore have a high level of awareness and integrity to cope with this disease and to control and manage the spread of the virus. Thus, this study aimed to evaluate the level of awareness and attitude of dentists related to COVID-19 and infection control.

\section{MATERIAL AND METHODS}

\section{Study sample}

The study sample consisted of dentistry students (year-5), dentists, and specialist dentists, in Turkey, regardless of the place of work. The electronic addresses of the samples were obtained from the Turkish Dental Association (20000 e-mails), and their presence was assessed by confirming their places of work and registration with this association. Within all samples, 10040 dentists/dentistry students were randomly chosen to participate in the present study. Each randomly selected participant was also contacted individually to make sure they were a dentistry student/dentist and were working in Turkey.

An online questionnaire form was created using Google Forms for data collection, and this questionnaire was sent to the previously selected emails. To test the questionnaire's comprehensibility, a pilot study was conducted with 40 dentists who were randomly selected. Cronbach's alpha score was calculated as 0.78 , and findings of the pilot study were not included to the study. Each participant was clearly informed about the aim and content of the study. The submission of the questionnaire implied their full consent. The study was approved by the Ethics Committee of the Istanbul University, Faculty of Medicine (protocol number 2020/15) and was conducted between 29th April 2020 and 29th May 2020.

\section{Questionnaire design}

The present study was performed using self-administered structured questionnaires. The questionnaire comprised 9 questions grouped into 5 domains: 1) demographic and professional characteristics, 2) knowledge level of contamination, 3) risk assessment of dental applications, 4) survival time of SARS-CoV-2 assessment, and 5) attitudes toward treating 
patients with COVID-19. Questionnaire consisted of yes/no and multiple-choice questions. All questions had to be answered to submit the survey and approximately ten minutes were required to fulfill it. Prior to the study, the questionnaire was pre-tested and required modifications were performed (Figure 1).

According to the domains and questions, the parameters used for the design of the questionnaire are below:

With Question 1, it was aimed to evaluate participants' levels of knowledge and considerations about the risk of COVID-19 contamination through saliva during related dental treatments.

With Question 2, frequently used dental applications, even in urgent cases, were specified and participants were asked to evaluate the risk levels of these treatment types.

Questions 3, 4, 5, 6, and 7, it was aimed to determine whether the participants were aware of the current data about the viability of COVID-19 on different surfaces (plastic, stainless steel, etc.) and aerosol.

With Question 8, the opinions about the effect of coughing or sneezing, which are part of daily life, on aerosol generation were aimed to evaluate.

Question 9 aimed to evaluate the attitude of the participants regarding the plexiglass box called the Houston Methodist Aerosol Container, which is used for protective purposes during the treatment of patients with COVID-19 in this pandemic period [25].

COVID-19 (Coronavirus Disease 2019) Awareness and Attitude Among Dentists

1. Which branch of dentistry has the highest risk of saliva contamination in COVID-19?

- Oral and Maxillofacial Surgery

- Prosthodontics

- Restorative Dentistry

- Oral Radiology
- Endodontics

- Periodontics

- Pediatric Dentistry

- Orthodontics

2.What is the risk of COVID-19 contamination of the following applications? (Number from 1 to 5 ; 1 : No risk, 5 : Highest risk)

\begin{tabular}{|c|c|c|c|c|c|}
\hline Treatment type & 1 & 2 & 3 & 4 & 5 \\
\hline \multicolumn{6}{|l|}{ Intraoral examination } \\
\hline \multicolumn{6}{|l|}{ Taking impression with alginate } \\
\hline \multicolumn{6}{|l|}{ Tooth preparation } \\
\hline \multicolumn{6}{|l|}{ Abrading painful dentures } \\
\hline \multicolumn{6}{|l|}{$\begin{array}{c}\text { Transportation of dental laboratory } \\
\text { works }\end{array}$} \\
\hline \multicolumn{6}{|l|}{ Scaling and root planing } \\
\hline \multicolumn{6}{|l|}{ Bracket placement } \\
\hline \multicolumn{6}{|l|}{ Tooth extraction } \\
\hline \multicolumn{6}{|l|}{ Pulpectomy } \\
\hline Filling & & & & & \\
\hline Obtaining intraoral radiograph & & & & & \\
\hline
\end{tabular}

3. How many hours does the virus survive in the infectious form on the plastic surfaces?
A) 72 hours
B) 48 hours
C) 24 hours

4. How many hours does the virus survive in the infectious form on stainless steel?
A) 72 hours
B) 48 hours
C) 24 hours

5. How many hours does the virus survive in the infectious form on cardboard or paper?
A) 72 hours
B) 48 hours
C) 24 hours

6. How many hours does the virus survive in the infectious form on copper?
A) 12 hours
B) 6 hours
C) 4 hours aerosols?

7. How many hours does the virus remain viable in
A) 6 hours
B) 4 hours
C) 3 hours 
8. Do you think the droplets that occur during coughing or sneezing form aerosols?

A) Yes

B) No

9. What are your thoughts on performing intraoral treatment procedures in the following plexiglass box?

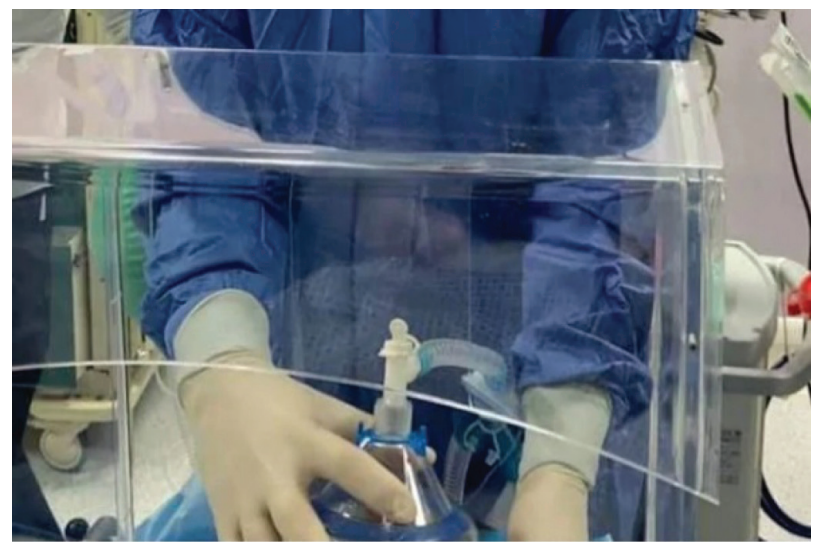

Figure 1- Questionnaire form of the study.

- I think this box will be useful to reduce transmission, I prefer to use it.

- I think this box will be useful to reduce transmission, but I do not prefer to use it.

- I do not think that this box will play a role in transmission, I prefer to apply treatment with protective equipment.

\section{Data extraction and statistical analysis}

All data were computerized in a dedicated database. The data were analyzed using IBM SPSS V23. Chi-square test was used to compare categorical data. Categorical data were presented as frequency (percent). The significance level was set at $\mathrm{p} \leq 0.05$ and missing data were not considered.

\section{RESULTS}

\section{Demographic characteristics of the sample population}

The questionnaire form was sent to 10000 dentists and dentistry students in Turkey, and a total of 3923 participants agreed to participate to the present study by submitting this form. 98 of these submitted questionnaires were excluded from the study, as these forms were partially filled or unfilled. Thus, 3825 final participants were included in the study, the age of participants ranged from 21-54 years with a mean of 29.1 \pm 7.6 years (Table I).

Table I - Frequency distributions according to demographic information of the participants

\begin{tabular}{|c|c|c|}
\hline & Frequency (n) & Percent (\%) \\
\hline \multicolumn{3}{|l|}{ Gender } \\
\hline Female & 2403 & 62.8 \\
\hline Male & 1422 & 37.2 \\
\hline \multicolumn{3}{|l|}{ Age } \\
\hline $18-30$ years & 2475 & 64.7 \\
\hline $31-44$ years & 1071 & 28.0 \\
\hline 45-years & 279 & 7.3 \\
\hline \multicolumn{3}{|l|}{ Professional status } \\
\hline Dentistry student & 1503 & 39.3 \\
\hline Graduate dentist & 1494 & 39.1 \\
\hline Prosthodontist & 351 & 9.2 \\
\hline Pediatric dentist & 135 & 3.5 \\
\hline Ortodontist & 126 & 3.3 \\
\hline Oral surgeon & 81 & 2.1 \\
\hline Periodontist & 54 & 1.4 \\
\hline Restorative dentist & 27 & 0.7 \\
\hline Oral radiologist & 27 & 0.7 \\
\hline Endodontist & 27 & 0.7 \\
\hline
\end{tabular}

Evaluation of the risk of saliva contamination of dental branches

Periodontics was the most frequent response to the question of which branch of dentistry poses the highest risks of saliva contamination in COVID-19 (32.2\%). Participants answered Orthodontics as the least risky dentistry branch with a rate of $0.2 \%$ (Table II). (Additional data were presented as supplementary files (Supplemental Table-I and II)). 
Table II - Comparison of risk assessment of dental branches regarding saliva contamination in COVID-19

\begin{tabular}{|c|c|c|c|c|}
\hline & $\begin{array}{c}\text { Female } \\
(n=2403)\end{array}$ & $\begin{array}{c}\text { Male } \\
(n=1422)\end{array}$ & $\begin{array}{c}\text { Test } \\
\text { statistics }\end{array}$ & $p$ \\
\hline $\begin{array}{l}\text { Which branch of dentistr } \\
\text { has the highest risk of } \\
\text { saliva contamination in } \\
\text { COVID-19? }\end{array}$ & & & & \\
\hline Periodontics & $855(35.6)^{\star}$ & $378(26.6)$ & \multirow{8}{*}{$x^{2}=6.373$} & \multirow{8}{*}{0.497} \\
\hline Restorative Dentistry & 432 (18) & $261(18.4)$ & & \\
\hline Prosthodontics & $360(15)$ & $261(18.4)$ & & \\
\hline Endodontics & $360(15)$ & $252(17.7)$ & & \\
\hline Oral\&Maxillofacial Surgery & $279(11.6)$ & $189(13.3)$ & & \\
\hline Pediatric Dentistry & $81(3.4)$ & $36(2.5)$ & & \\
\hline Oral Radiology & $36(1.5)$ & $36(2.5)$ & & \\
\hline Orthodontics & $0(0)$ & $9(0.6)$ & & \\
\hline
\end{tabular}

$\mathrm{X}^{2}$ : Chi-square test statistics ${ }^{*}$ Frequency (percent)

Evaluation of the risk of COVID-19 contamination regarding dental application

The frequencies of the scores that the participants gave to the COVID-19 transmission risk of dental applications are displayed in Figure 2. Dental treatment applications that are scored mainly with 5 in the risk assessment and considered the riskiest procedures are the following: tooth preparation (69.4\%), scaling and root planing (63.5\%), filling (53.4\%), and pulpectomy (40.5\%). According to the results, it is seen that all dentistry applications are considered to carry risks at varying degrees. (Additional data were presented as supplementary files (Supplemental Table-III and IV)).

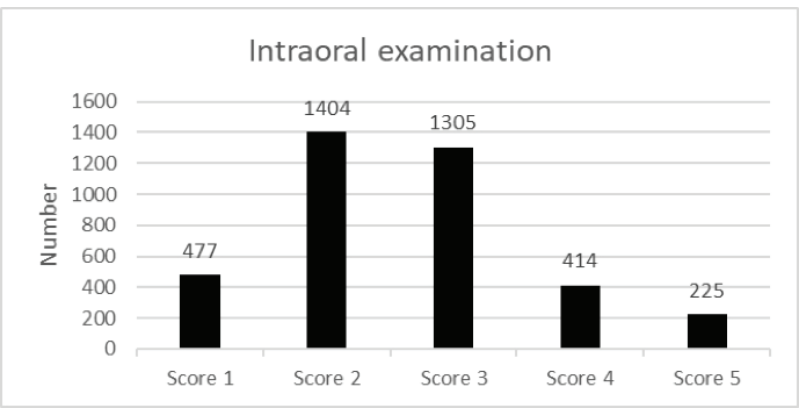

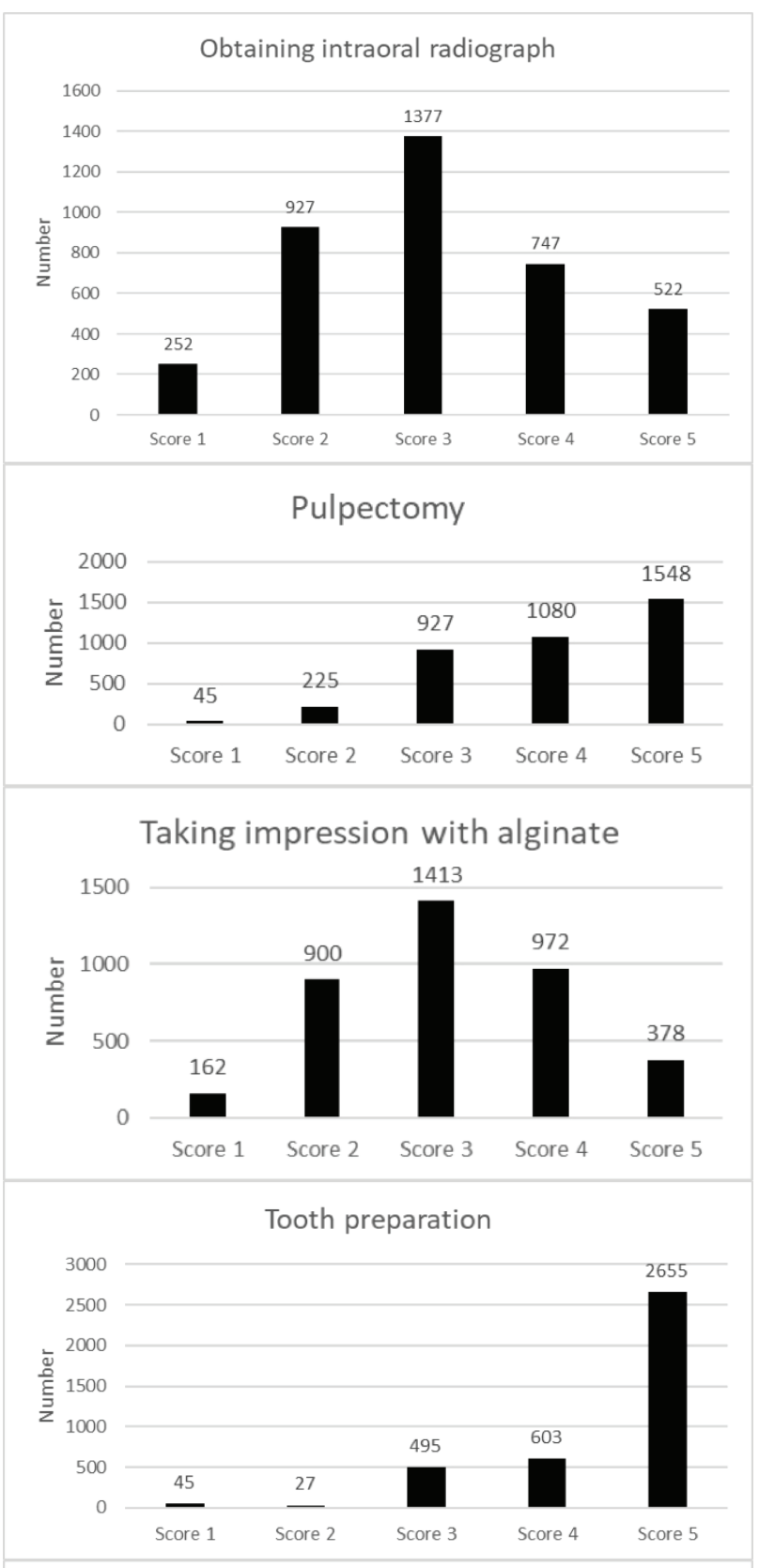

Abrading painful dentures

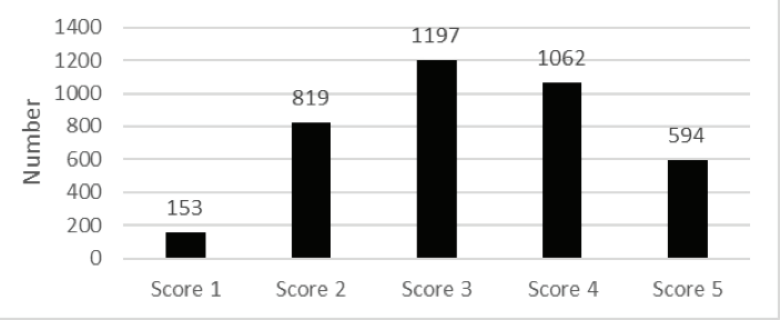




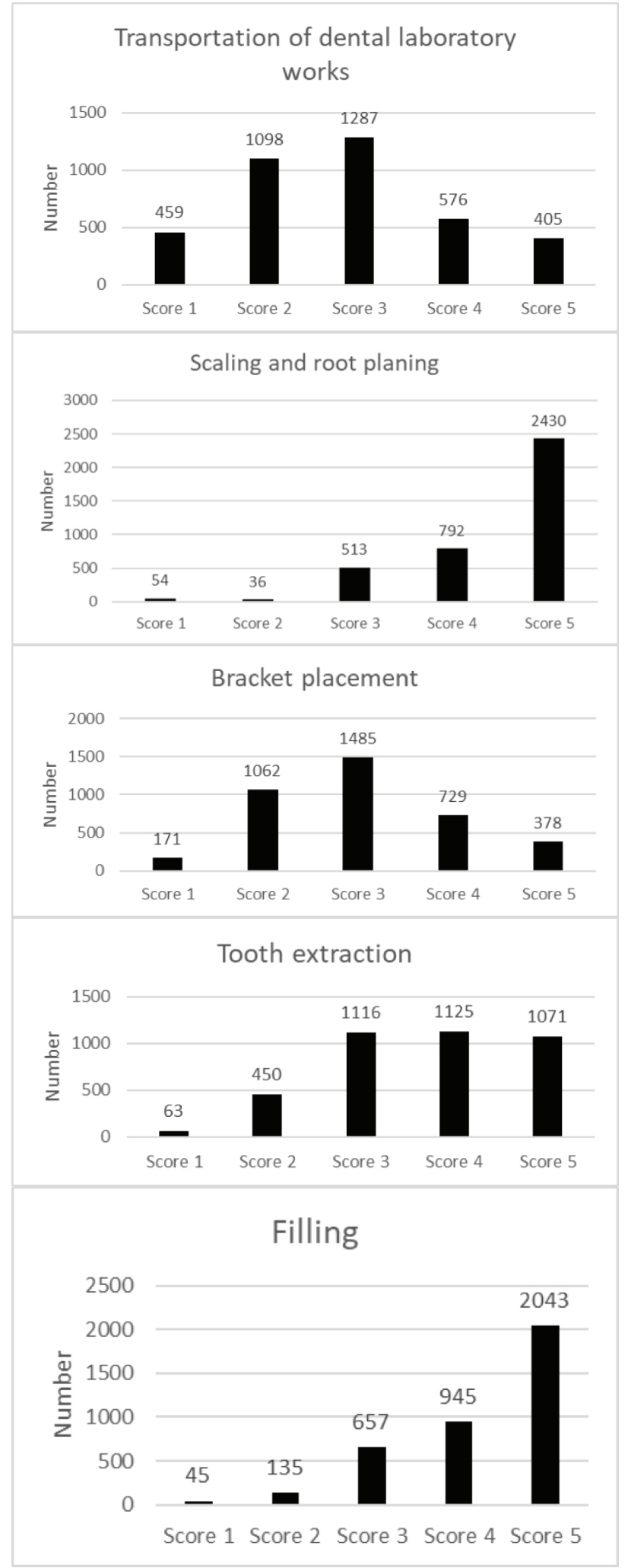

Figure 2 - Frequency distributions of the dental applications according to the scores given to COVID-19 contamination risk (1: No risk, 5: Highest risk).
Table III - Comparison of the scores given to the dental applications regarding SARS-CoV-2 contamination risk by gender (1: No risk, 5: Highest risk)

\begin{tabular}{|c|c|c|c|c|}
\hline & $\begin{array}{c}\text { Female } \\
(n=2403)\end{array}$ & $\begin{array}{c}\text { Male } \\
(n=1422)\end{array}$ & $\begin{array}{c}\text { Test } \\
\text { statistics }\end{array}$ & $p$ \\
\hline \multicolumn{5}{|c|}{ Intraoral examination } \\
\hline Score 1 & $288(12)^{*}$ & $189(13.3)$ & \multirow{5}{*}{$X^{2}=11.116$} & \multirow{5}{*}{0.025} \\
\hline Score2 & $756(31.5)$ & $648(45.6)$ & & \\
\hline Score 3 & $891(37.1)$ & $414(29.1)$ & & \\
\hline Score 4 & $315(13.1)$ & $99(7)$ & & \\
\hline Score 5 & $153(6.4)$ & $72(5.1)$ & & \\
\hline \multicolumn{5}{|c|}{ Taking impression with alginate } \\
\hline Score 1 & $90(3.7)$ & $72(5.1)$ & \multirow{5}{*}{$x^{2}=7.873$} & \multirow{5}{*}{0.096} \\
\hline Score 2 & $477(19.9)$ & $423(29.7)$ & & \\
\hline Score 3 & $891(37.1)$ & $522(36.7)$ & & \\
\hline Score 4 & $684(28.5)$ & $288(20.3)$ & & \\
\hline Score 5 & $261(10.9)$ & $117(8.2)$ & & \\
\hline \multicolumn{5}{|c|}{ Tooth preparation } \\
\hline Score1 & $18(0.7)$ & $27(1.9)$ & \multirow{5}{*}{$x^{2}=6.425$} & \multirow{5}{*}{0.170} \\
\hline Score2 & $18(0.7)$ & $9(0.6)$ & & \\
\hline Score 3 & $315(13.1)$ & $180(12.7)$ & & \\
\hline Score 4 & $306(12.7)$ & $297(20.9)$ & & \\
\hline Score 5 & $1746(72.7)$ & $909(63.9)$ & & \\
\hline \multicolumn{5}{|c|}{ Abrading painful dentures } \\
\hline Score 1 & $117(4.9)$ & $36(2.5)$ & \multirow{5}{*}{$X^{2}=5.481$} & \multirow{5}{*}{0.241} \\
\hline Score2 & $459(19.1)$ & $360(25.3)$ & & \\
\hline Score 3 & $711(29.6)$ & $486(34.2)$ & & \\
\hline Score 4 & $720(30)$ & $342(24.1)$ & & \\
\hline Score 5 & $396(16.5)$ & $198(13.9)$ & & \\
\hline \multicolumn{5}{|c|}{ Transportation of dental laboratory works } \\
\hline Score1 & $297(12.4)$ & $162(11.4)$ & & \\
\hline Score 2 & $585(24.3)$ & $513(36.1)$ & & \\
\hline Score 3 & $918(38.2)$ & $369(25.9)$ & $x^{2}=9.422$ & 0.051 \\
\hline Score 4 & $360(15)$ & $216(15.2)$ & & \\
\hline Score 5 & $243(10.1)$ & $162(11.4)$ & & \\
\hline Scaling and rc & & & & \\
\hline Score 1 & $27(1.1)$ & $27(1.9)$ & & \\
\hline Score2 & $27(1.1)$ & $9(0.6)$ & & \\
\hline Score 3 & $315(13.1)$ & $198(13.9)$ & $x^{2}=3.510$ & 0.476 \\
\hline Score 4 & $441(18.4)$ & $351(24.7)$ & & \\
\hline Score 5 & 1593(66.3) & $837(58.9)$ & & \\
\hline Bracket place & & & & \\
\hline Score 1 & $117(4.9)$ & $54(3.8)$ & & \\
\hline Score 2 & $612(25.5)$ & $450(31.6)$ & & \\
\hline Score 3 & $936(39)$ & $549(38.6)$ & $x^{2}=3.019$ & 0.555 \\
\hline Score 4 & $468(19.5)$ & $261(18.4)$ & & \\
\hline Score 5 & $270(11.2)$ & $108(7.6)$ & & \\
\hline Tooth extract & & & & \\
\hline Score 1 & $27(1.1)$ & $36(2.5)$ & & \\
\hline Score2 & $315(13.1)$ & $135(9.5)$ & & \\
\hline Score 3 & $684(28.5)$ & $432(30.4)$ & $x^{2}=3.227$ & 0.521 \\
\hline Score 4 & $675(28.1)$ & $450(31.6)$ & & \\
\hline Score 5 & $702(29.2)$ & $369(25.9)$ & & \\
\hline Pulpectomy & & & & \\
\hline Score 1 & $27(1.1)$ & $18(1.3)$ & & \\
\hline Score2 & $117(4.9)$ & $108(7.6)$ & & \\
\hline Score 3 & $549(22.8)$ & $378(26.6)$ & $x^{2}=4.037$ & 0.401 \\
\hline Score 4 & $657(27.3)$ & $423(29.7)$ & & \\
\hline Score 5 & $1053(43.8)$ & $495(34.8)$ & & \\
\hline Filling & & & & \\
\hline Score 1 & $18(0.7)$ & $27(1.9)$ & & \\
\hline Score 2 & $81(3.4)$ & $54(3.8)$ & & \\
\hline Score 3 & $396(16.5)$ & $261(18.4)$ & $x^{2}=5.384$ & 0.250 \\
\hline Score 4 & $531(22.1)$ & $414(29.1)$ & & \\
\hline Score 5 & $1377(57.3)$ & $666(46.8)$ & & \\
\hline Obtaining int & graph & & & \\
\hline Score 1 & $207(8.6)$ & $45(3.2)$ & & \\
\hline Score2 & $504(21)$ & $423(29.7)$ & & \\
\hline Score 3 & $765(31.8)$ & $612(43)$ & $x^{2}=17.635$ & 0.001 \\
\hline Score 4 & $558(23.2)$ & $189(13.3)$ & & \\
\hline Score 5 & $369(15.4)$ & $153(10.8)$ & & \\
\hline
\end{tabular}

$\mathrm{X}^{2}$ : Chi-square test statistics ${ }^{*}$ Frequency (percent) 
Table IV - Comparison of the scores given to the dental applications regarding COVID-19 contamination risk by professional status (1: No risk, 5: Highest risk)

\begin{tabular}{|c|c|c|c|c|c|}
\hline & $\begin{array}{c}\text { Dentistry } \\
\text { student } \\
(n=1503)\end{array}$ & $\begin{array}{c}\text { Graduate } \\
\text { dentist } \\
(n=1494)\end{array}$ & $\begin{array}{c}\text { Specialist } \\
\text { dentist } \\
(n=828)\end{array}$ & $\begin{array}{c}\text { Test } \\
\text { statistics }\end{array}$ & $p$ \\
\hline \multicolumn{6}{|c|}{ Intraoral examination } \\
\hline Score 1 & $180(12)^{*}$ & $189(127)$ & $108(13)$ & \multirow{5}{*}{$x^{2}=10.087$} & \multirow{5}{*}{0.259} \\
\hline Score 2 & $621(41.3)$ & $468(31.3)$ & $315(38)$ & & \\
\hline Score 3 & $441(29.3)$ & $540(36.1)$ & $324(39.1)$ & & \\
\hline Score 4 & $144(9.6)$ & $207(13.9)$ & $63(7.6)$ & & \\
\hline Score 5 & $117(7.8)$ & $90(6)$ & $18(2.2)$ & & \\
\hline \multicolumn{6}{|c|}{ Taking impression with alginate } \\
\hline Score 1 & $27(1.8)$ & $63(4.2)$ & $72(8.7)$ & \multirow{5}{*}{$x^{2}=11.457$} & \multirow{5}{*}{0.177} \\
\hline Score2 & $378(25.1)$ & $360(24.1)$ & $162(19.6)$ & & \\
\hline Score 3 & $513(34.1)$ & $549(36.7)$ & $351(424)$ & & \\
\hline Score 4 & $414(27.5)$ & $360(24.1)$ & $198(23.9)$ & & \\
\hline Score 5 & 171(11.4) & $162(10.8)$ & $45(5.4)$ & & \\
\hline \multicolumn{6}{|c|}{ Tooth preparation } \\
\hline Score 1 & $27(1.8)$ & $0(0)$ & $18(2.2)$ & \multirow{5}{*}{$x^{2}=16.432$} & \multirow{5}{*}{0.037} \\
\hline Score 2 & $0(0)$ & $18(1.2)$ & $9(1.1)$ & & \\
\hline Score 3 & $261(17.4)$ & $162(10.8)$ & $72(8.7)$ & & \\
\hline Score 4 & $297(19.8)$ & $225(15.1)$ & $81(9.8)$ & & \\
\hline Score 5 & $918(61.1)$ & 1089(72.9) & $648(78.3)$ & & \\
\hline \multicolumn{6}{|c|}{ Abrading painful dentures } \\
\hline Score 1 & $72(4.8)$ & $45(3)$ & $36(4.3)$ & \multirow{5}{*}{$x^{2}=9.216$} & \multirow{5}{*}{0.324} \\
\hline Score 2 & $360(24)$ & $333(22.3)$ & $126(15.2)$ & & \\
\hline Score 3 & $504(33.5)$ & $486(32.5)$ & $207(25)$ & & \\
\hline Score 4 & $369(24.6)$ & $387(25.9)$ & $306(37)$ & & \\
\hline Score 5 & $198(13.2)$ & $243(16.3)$ & $153(18.5)$ & & \\
\hline \multicolumn{6}{|c|}{ Transportation of dental laboratory works } \\
\hline Score 1 & $189(12.6)$ & $144(9.6)$ & $126(15.2)$ & & \\
\hline Score2 & $531(35.3)$ & $369(24.7)$ & $198(23.9)$ & & \\
\hline Score 3 & $495(32.9)$ & $540(36.1)$ & $252(30.4)$ & $x^{2}=12.259$ & 0.140 \\
\hline Score 4 & $144(9.6)$ & $279(18.7)$ & $153(18.5)$ & & \\
\hline Score 5 & $144(9.6)$ & $162(10.8)$ & $99(12)$ & & \\
\hline Scaling & root planir & & & & \\
\hline Score 1 & $36(2.4)$ & $0(0)$ & $18(2.2)$ & & \\
\hline Score 2 & $27(1.8)$ & $9(0.6)$ & $0(0)$ & & \\
\hline Score 3 & $198(13.2)$ & $216(14.5)$ & $99(12)$ & $x^{2}=7.187$ & 0.517 \\
\hline Score 4 & $324(21.6)$ & $315(211.1)$ & $153(18.5)$ & & \\
\hline Score 5 & $918(61.1)$ & $954(63.9)$ & $558(67.4)$ & & \\
\hline Bracket & ement & & & & \\
\hline Score 1 & $72(4.8)$ & $54(3.6)$ & $45(5.4)$ & & \\
\hline Score 2 & $522(34.7)$ & $351(23.5)$ & $189(22.8)$ & & \\
\hline Score 3 & $486(32.3)$ & $675(45.2)$ & $324(39.1)$ & $x^{2}=11.738$ & 0.163 \\
\hline Score 4 & $270(18)$ & $252(16.9)$ & $207(25)$ & & \\
\hline Score 5 & $153(10.2)$ & $162(10.8)$ & $63(7.6)$ & & \\
\hline Toothes & tion & & & & \\
\hline Score1 & $27(1.8)$ & $27(1.8)$ & $9(1.1)$ & & \\
\hline Score 2 & $180(12)$ & $126(8.4)$ & $144(17.4)$ & & \\
\hline Score 3 & $360(24)$ & $495(33.1)$ & $261(31.5)$ & $x^{2}=14.553$ & 0.068 \\
\hline Score 4 & $396(26.3)$ & $477(31.9)$ & $252(30.4)$ & & \\
\hline Score 5 & $540(35.9)$ & $369(24.7)$ & $162(19.6)$ & & \\
\hline Pulpect & & & & & \\
\hline Score 1 & $18(1.2)$ & $9(0.6)$ & $18(2.2)$ & & \\
\hline Score 2 & $135(9)$ & $45(3)$ & $45(5.4)$ & & \\
\hline Score 3 & $360(24)$ & $369(24.7)$ & $198(23.9)$ & $x^{2}=7.396$ & 0.495 \\
\hline Score 4 & $396(26.3)$ & $468(31.3)$ & $216(26.1)$ & & \\
\hline Score 5 & $594(39.5)$ & $603(40.4)$ & $351(424)$ & & \\
\hline Filling & & & & & \\
\hline Score 1 & $27(1.8)$ & $0(0)$ & $18(2.2)$ & & \\
\hline Score 2 & $72(4.8)$ & $54(3.6)$ & $9(1.1)$ & & \\
\hline Score 3 & 288(19.2) & $243(16.3)$ & $126(15.2)$ & $X^{2}=9.025$ & 0.340 \\
\hline Score 4 & $405(26.9)$ & $360(24.1)$ & $180(21.7)$ & & \\
\hline Score 5 & $711(47.3)$ & $837(56)$ & $495(59.8)$ & & \\
\hline Obtaini & traoral rad & graph & & & \\
\hline Score 1 & $90(6)$ & $90(6)$ & $72(8.7)$ & & \\
\hline Score2 & $414(27.5)$ & $324(21.7)$ & $189(22.8)$ & & \\
\hline Score 3 & $486(32.3)$ & 621(41.6) & $270(32.6)$ & $x^{2}=7.507$ & 0.483 \\
\hline Score 4 & $261(17.4)$ & 297 (19.9) & $189(22.8)$ & & \\
\hline Score 5 & $252(16.8)$ & $162(10.8)$ & $108(13)$ & & \\
\hline
\end{tabular}

$\mathrm{X}^{2}$ : Chi-square test statistics * Frequency (percent)

\section{Evaluation of the virus survival time by} surface

Figure 3 shows that most participants knew the survival time of SARS-CoV-2 on plastic (45.9\%) and cardboard/paper (46.1\%) surfaces, while they knew less about the survival time for stainless steel (38.1\%) and copper surfaces (16.2\%). (Additional data were presented as Supplementary Table V).

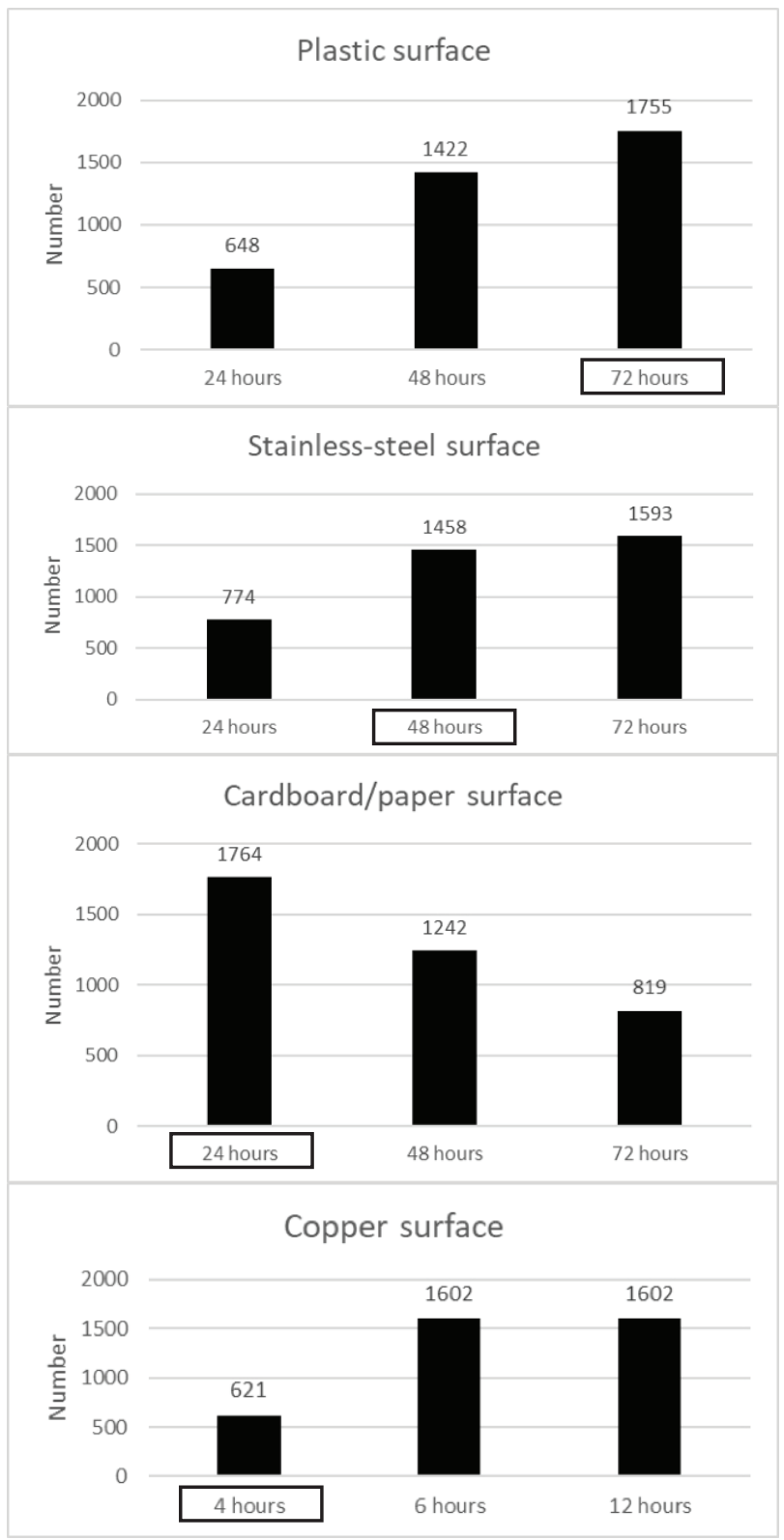

Figure 3 - Frequency distributions of the survival time of SARS-CoV-2 regarding different surface types.

*The circle under the relevant bar indicates the virus viability time for each surface. 


\section{Aerosol evaluation}

The participants answered the question predominantly correctly, which allowed evaluating daily activities, such as coughing and sneezing, regarding aerosol-forming. However, 33.4\% of them knew the survival time of the virus in the aerosol (Figure 4). (Additional data were presented as supplementary file-Supplementary Table V).

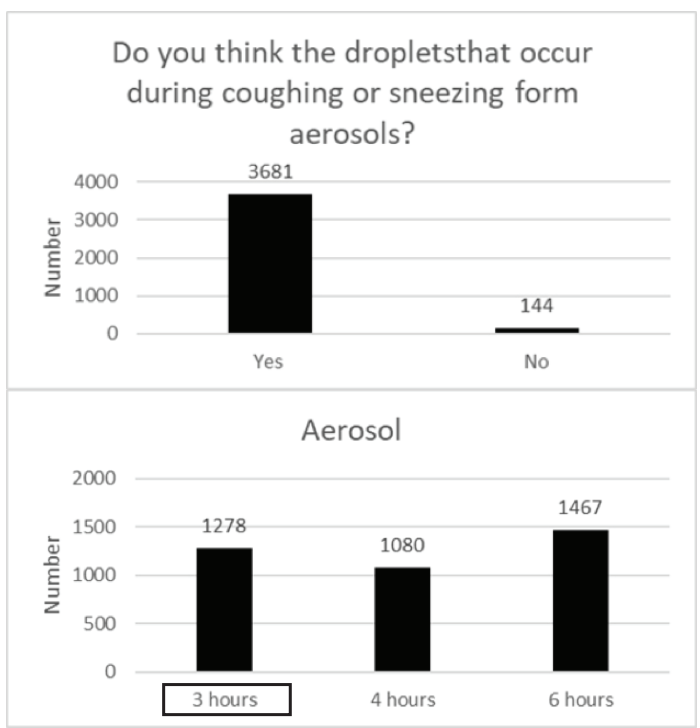

Figure 4 - Frequency distributions of aerosol-forming activities and the survival time of SARS-CoV-2 in the aerosol.

*The circle under the relevant bar indicates the virus viability time for aerosol.

Table $\mathbf{V}$ - Comparison of the survival time of SARS-CoV-2 regarding different surface types and aerosol form by gender

\begin{tabular}{|c|c|c|c|c|}
\hline & $\begin{array}{c}\text { Female } \\
(n=2403)\end{array}$ & $\begin{array}{c}\text { Male } \\
(n=1422)\end{array}$ & $\begin{array}{c}\text { Test } \\
\text { statistics }\end{array}$ & $p$ \\
\hline \multicolumn{5}{|c|}{ Plastic surface } \\
\hline 24 hours & $378(15.7)^{\star}$ & $270(19)$ & \multirow{3}{*}{$x^{2}=21.785$} & \multirow{3}{*}{$<0.001$} \\
\hline 48 hours & $720(30)$ & $702(49.4)$ & & \\
\hline 72 hours & $1305(54.3)$ & $450(31.6)$ & & \\
\hline \multicolumn{5}{|c|}{ Stainless steel surface } \\
\hline 24 hours & $486(20.2)$ & $288(20.3)$ & \multirow{3}{*}{$x^{2}=0.162$} & \multirow{3}{*}{0.922} \\
\hline 48 hours & $900(37.5)$ & $558(39.2)$ & & \\
\hline 72 hours & $1017(42.3)$ & $576(40.5)$ & & \\
\hline \multicolumn{5}{|c|}{ Cardboard or paper surface } \\
\hline 24 hours & $1134(47.2)$ & $630(44.3)$ & \multirow{3}{*}{$x^{2}=3.115$} & \multirow{3}{*}{0.211} \\
\hline 48 hours & $711(29.6)$ & $531(37.3)$ & & \\
\hline 72 hours & $558(23.2)$ & 261(18.4) & & \\
\hline \multicolumn{5}{|c|}{ Copper surface } \\
\hline 4 hours & $432(18)$ & $189(13.3)$ & \multirow{3}{*}{$x^{2}=1.711$} & \multirow{3}{*}{0.425} \\
\hline 6 hours & $972(40.4)$ & $630(44.3)$ & & \\
\hline 12 hours & $999(41.6)$ & $603(42.4)$ & & \\
\hline \multicolumn{5}{|l|}{ Aerosol } \\
\hline 3 hours & $756(31.5)$ & $522(36.7)$ & \multirow{3}{*}{$x^{2}=1.351$} & \multirow{3}{*}{0.509} \\
\hline 4 hours & $711(29.6)$ & $369(25.9)$ & & \\
\hline 6 hours & $936(39)$ & $531(37.3)$ & & \\
\hline \multicolumn{5}{|c|}{$\begin{array}{l}\text { Do you think the droplets that occur during coughing or sneezing } \\
\text { form aerosols? }\end{array}$} \\
\hline Yes & 2313(96.3) & $1368(96.2)$ & \multirow[b]{2}{*}{$x^{2}=0.001$} & \multirow[b]{2}{*}{0.978} \\
\hline No & $90(3.7)$ & $54(3.8)$ & & \\
\hline
\end{tabular}

$\mathrm{X}^{2}$ : Chi-square test statistics * Frequency (percent)

\section{Plexiglass box preference}

More than half of the participants reported that the plexiglass box in the questionnaire form is useful to reduce transmission of COVID-19 and they prefer to use it during dental treatment (Figure 5).

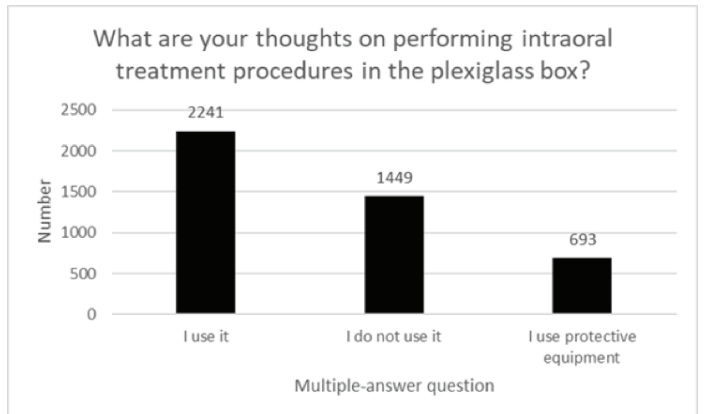

Figure 5 - Frequency distributions of plexiglass box preference during dental treatment.

\section{DISCUSSION}

This cross-sectional study was conducted to evaluate Turkish dentists' awareness and attitude levels related to COVID-19 and SARSCoV-2.

Salivary gland epithelial cells can be infected by SARS-CoV, a type of virus similar to SARS-CoV-2 / COVID-19, and may be the main source of this virus in saliva and SARS-CoV-2 can be found in asymptomatic patients for up to 29 days $[6,26]$. This is of clinical importance for the dental team due to the reporting of recurrence during the convalescence period and even after patient's recovery [27]. At some point, a confirmed virus carrier may need urgent dental treatment and knowing the risks associated with dental procedures is of importance for the prevention of transmission [10]. In this study, $32.2 \%$ of Turkish dentists considered Periodontics as associated with the highest risk of SARS-CoV-2 saliva contamination. When compared with a recent study, this result was inconsistent, as $83.1 \%$ of the participants agreed to perform periodontal treatments, such as a periodontal abscess or oral mucosa lesions [28]. This may be due to 
evaluated treatment types were emergent by being acute and/or painful. The response to this question differed depending on professional status as most of the specialist dentists (27.2\%) thought that Prosthodontics was the riskiest practice $(p=0.022)$. This result may be due to the high proportion of prosthodontists in our specialist dentist population, as significant relationship between specialty and knowledge level was stated in the literature [29-32].

Aerosol is defined as a suspension of liquid or solid particles in the air produced by animals, humans, machines, or instruments and consists of tiny particles called droplets ( $>$ $5 \mu \mathrm{m})$ or droplet nuclei $(1 \pm 5 \mu \mathrm{m})$ [24]. Aerosol has been found to be the primary route for COVID-19 transmission and droplets have been proved to contaminate surfaces within 1 meter $[13,33]$. Considering this situation, the risk of COVID-19 transmission is significantly high in a treatment that requires face-to-face contact, such as dentistry. The type of aerosol formed in particles of organisms is called bioaerosol, and dentistry is rich in this type of aerosol due to the use of air turbines, high-speed handpieces, ultrasonic scalers, air-water syringes, and three in one syringe [24]. Coughing, sneezing, talking, and breathing are other factors that cause the formation of bioaerosol [34]. In this study, almost all participants (96.2\%) displayed their awareness about virus transmission by approving that coughing or sneezing form aerosols. In line with the findings of this research, studies in the literature reported that most dentists were aware of the transmission routes of this virus [2,29-31,35].

It is also important even after the completion of the treatment as aerosols remain in the air for a long time [36]. To prevent transmission, fortified personal protective gear like disposable caps, gloves, surgical masks, scrubs, protective face/ eye shields, and impermeable shoe cover was adopted as biosafety precautions with additional recommendations about the dental environment, chairside applications, and patients' appointments, due to this pandemic $[18,37]$. With this aim, it has also been reported that aerosol-generating procedures should be avoided [6]. In addition to aerosol generation, aerosol contamination is also important for the transmission of the COVID-19 in dental practice as position and distance in dental operation have been found to be effective in the dissemination of aerosol [36]. The results of this study showed that $33.4 \%$ of the participants were aware of the viability time of this virus in the aerosol (3 hours) and 38.4\% responded 6 hours. This reveals that the information needs to be updated when we consider the role of aerosol in dental practice. Since this is the first study to evaluate the knowledge level about the viability time of this virus in the aerosol, a direct comparison could not be made in the literature.

In preventing the dissemination of aerosols, rubber dam has been reported to play an effective role with good patient acceptance [38]. In a recent international study about COVID-19, $84 \%$ of the dentists reported not using rubber dam isolation for every patient [2]. Being different from this study, we evaluated the attitude towards the use of plexiglass box to control the spread of the virus during dental treatment, and more than half of the participants $(58.7 \%)$ reported that they would benefit from this tool. When the attitude towards surgical masks is compared for protective reasons in the literature, approximately $90 \%$ of dentists stated that these masks cannot provide sufficient protection [2,28]. Thus, the rate of box preference resulted in the present study may be explained by this common tendency among dentists. Although the plexiglass box can reduce the vision of the dentist during treatment and lead to disadvantages in claustrophobic patients, the findings of the present study shows that dentists seem open 
to new precaution methods and aware of new actions due to COVID-19.

Throughout this pandemic, the necessity for the classification of dental treatments according to the degree of risk has been suggested in recent literature [6]. Therefore, the risk of COVID-19 contamination has been evaluated in this study by including 11 frequently performed dental applications. Tooth preparation, scaling and root planing, filling, and pulpectomy were considered the riskiest dental applications. This can be attributed that these dental applications are more prone to aerosol-generating than others, as ultrasonic scaler has been indicated to be the greatest producer of aerosol [33]. Tooth preparation (69.4\%) was the riskiest among all dental applications, and the score for the tooth preparation was significantly different between groups depending on the professional status with the highest rate from specialist dentists (78.3\%). Since the number of prosthodontists was high in this study, it could induce a bias in this observation. Although Periodontics was considered the highest risk of saliva contamination in COVID-19, participants gave 5 for tooth preparation in the risk assessment of dental applications. This may be due to the fact that the parameter being evaluated (tooth preparation) was specified as dental application rather than the main dentistry branch like Periodontics, and the formation of aerosol is inevitable due to the high-speed equipment needed in tooth preparation, while scaling and root planning can be performed with handpieces. Abrading the painful dentures causes aerosol generation, however, this application was interestingly scored 3 .

In a study evaluating dentists' attitudes towards dental applications, $13.9 \%$ of the participants stated that they could perform routine dental treatments when necessary precautions were taken, while $93.3 \%$ stated that they could perform only emergency procedures.
It was also stated by the participants that they would perform dental applications that do not create aerosol, with a rate of $40.4 \%$ [28]. In line with the findings of this study, high risk scores of the evaluated dental treatments given by our participants have been supported.

It is necessary to know the viability period of COVID-19 on the different types of surfaces because of the risk of aerosol exposure in the dental clinic [7]. According to the results, the surfaces included in this study and their known rates by participants are as follows: plastic (45.9\%), cardboard/paper (46.1\%), stainless steel (38.1\%), and copper (16.2\%). Compared to other studies in the literature, in our study, the awareness and knowledge level of the participants about the viability of the virus was found to be lower $[8,28,29,31]$. While evaluating more specific surfaces in our study, the evaluation of the virus incubation period, which is more general, in these studies can be justified for this result. However, this study is important for dentists to learn detailed information as well as general information about this virus due to ongoing pandemic.

The choice of the viability period of COVID-19 on the stainless-steel surface was different depending on the professional status. Interestingly, specialist dentists (25\%) answered this question less correctly than dentistry students (39.5\%) and graduate dentists (44\%). This result was inconsistent with the study which reported higher knowledge levels for specialist dentists [28]. From this result, it is seen that specialist dentists should update their knowledge. Besides the surface type, the humidity of the environment also affects the activity of this virus, as it shows increased activity in a humid environment. Thus, it is recommended to keep surfaces in the dental clinic dry and clean to prevent COVID-19 transmission. Although there is scarce data in the literature, COVID-19 is thought to be vulnerable to disinfectants 
such as $62-71 \%$ ethanol, $0.5 \%$ hydrogen peroxide, $0.1 \%$ sodium hypochlorite, phenolic and quaternary ammonium compounds [7].

Limitations of this study are the collection of data due to the rapid effect of the outbreak and low response rate of the survey, that resulted in smaller sample size than expected. Furthermore, respondents were only from Turkey and the study did not include all countries affected by this virus. Therefore, the present study has limited generalizability and the results cannot be globalized.

\section{CONCLUSIONS}

Within the limitations of this study, Periodontology has been reported to have the highest risk of saliva contamination in COVID-19. Tooth preparation, scaling and root planing, filling, and pulpectomy have been scored by 5 in the risk assessment of the included dental treatment applications. The results show that dentists or dentistry students need to do more to keep their knowledge and awareness up to date to mitigate the spread of COVID-19.

\section{Conflict of Interest}

The authors declare that they have no conflict of interest.

\section{Funding}

This study was partially supported by the Fulbright USA Commission under Grant Number of FY-2020-TR-PD-02.

\section{REFERENCES}

1. Meng L, Hua F, Bian Z. Coronavirus Disease 2019 (COVID-19): Emerging and future challenges for dental and oral medicine. J Dent Res 2020;99(5):4817. doi:10.1177/0022034520914246.

2. Ahmed MA, Jouhar R, Ahmed N, Adnan S, Aftab M, Zafar MS, et al. Fear and practice modifications among dentists to combat novel Coronavirus Disease (COVID-19) outbreak. Int J Environ Res Public Health 2020;17(8):2821. doi: 10.3390/ijerph17082821.
3. World Health Organization Coronavirus disease (COVID-19) pandemic. [Internet] Avaiable at: https://wwwwwho.int/emergencies/diseases/novelcoronavirus-2019. [Accessed 19 January 2021].

4. Machado RA, de Souza NL, Oliveira RM, Junior HM, Bonan PRF. Social media and telemedicine for oral diagnosis and counselling in the COVID-19 era. Oral Oncol 2020; 105:104685. doi: 10.1016/j.oraloncology.2020.104685.

5. Hillen HS, Kokic G, Farnung L, Dienemann C, Tegunov D, Cramer P. Structure of replicating SARS-CoV-2 polymerase. Nature. 2020 Aug;584(7819):154-6. doi: 10.1038/s41586-020-2368-8.

6. Alharbi A, Alharbi S, Alqaidi S. Guidelines for dental care provision during the COVID-19 pandemic. Saudi Dent J. 2020 May;32(4):181-6. doi: 10.1016/j. sdentj.2020.04.001.

7. Fallahi HR, Keyhan SO, Zandian D, Kim SG, Cheshmi B. Being a front-line dentist during the Covid-19 pandemic: a literature review. Maxillofac Plast Reconstr Surg. 2020 Apr 24;42(1):12. doi: 10.1186/s40902-020-00256-5.

8. Khader Y, Al Nsour M, Al-Batayneh OB, Saadeh R, Bashieer H, Alfaqih M, et al. Dentists' awareness, perception, and attitude regarding COVID-19 and infection control: Cross-sectional study among Jordanian dentists. JMIR Public Health Surveill 2020;6(2):e18798. doi: 10.2196/18798.

9. van Doremalen N, Bushmaker T, Morris DH, Holbrook MG, Gamble A, Williamson BN, et al. Aerosol and surface stability of SARS-CoV-2 as compared with SARS-CoV-1. N Engl J Med 2020;382(16):1564 7. doi: 10.1056/NEJMc2004973.

10. To KK, Tsang OT, Chik-Yan Yip C, Chan KH, Wu TC,Chan JMC, et al. Consistent detection of 2019 novel coronavirus in saliva. Clin Infect Dis. 2020 Jul 28;71(15):841-3. doi: 10.1093/cid/ciaa149.

11. Ministry of Health. [Internet]. Turkey [cited 2021 Jan 02]. Available from: https://www.saglik.gov.tr/.

12. Centers for Disease Control and Prevention. Morbidity and Mortality Weekly Report (MMWR) [Internet]. [cited 2021 Jan 10]. Available from: https://www.cdc.gov/mmwr/volumes/69/wr/mm6938a3.htm

13. Lai J, Ma S, Wang Y, Z Cai, J Hu, N Wei, et al. Factors associated with mental health outcomes among health care workers exposed to Coronavirus Disease 2019. JAMA Netw Open. 2020 Mar 2;3(3):e203976. doi: 10.1001/jamanetworkopen.2020.3976.

14. The American Dental Association. Summary of ADA Guidance During the COVID-19 Crisis. Available at: https://www.ada.org/en/press-room/ news-releases/2020-archives/april/summary-of-ada-guidance-duringthe-covid-19-crisis [Accessed 10 January 2021]

15. Centers for Disease Control and Prevention. Infection prevention \& control in dental settings [Internet]. USA [cited 2021 Jan 10]. Available from: https://www.cdc.gov/oralhealth/infectioncontrol/index.html.

16. FDI World Dental Federation. Dental practice and treatment guidance [Internet]. Geneva [cited 2021 Jan 10]. Available from: https://www. fdiworlddental.org/fdi-covid-19-resource-library.

17. Public Health England (PHE). COVID-19: infection prevention and control dental appendix [Internet]. UK [cited 2021 Jan 10]. Available from: https:// www.gov.uk/government/publications/wuhan-novel-coronavirusinfection-prevention-and-control.

18. Kilıcarslan MA, Senel Cizmeci F, Ozcan M. Assessment of dental care during the COVID-19 pandemic in Turkey and future projections. Brazil Dent Sci 2020;23(2): 1-7.doi: 10.14295/bds.2020.v23i2.2260.

19. Oztürk M, Ozeç I, Kiliç E. Utilisation of personal protective equipment in dental practice. Int Dent J. 2003 Aug;53(4):216-9. doi: 10.1111/j.1875595x.2003.tb00748.x 
20. Cirillo N. COVID-19 outbreak: succinct advice for dentists and oral healthcare professionals. Clin Oral Investig. 2020 Jul;24(7):2529-35. doi: 10.1007/s00784-020-03323-3.

21. British Dental Association (BDA). https://bda.247lib.com/bdaLib/ frm247CatDetails.aspx [Accessed 10 January 2021].

22. Ather A, Patel B, Ruparel NB, Diogenes A, Hargreaves KM. Coronavirus Disease 19 (COVID-19): Implications for clinical dental care. J Endod. 2020 May;46(5):584-95. doi:10.1016/j.joen.2020.03.008.

23. Guo H, Zhou Y, Liu X, Tan J. The impact of the COVID-19 epidemic on the utilization of emergency dental services. J Dent Sci. 2020 Dec;15(4):56467. doi: 10.1016/j.jds.2020.02.002.

24. Zemouri C, de Soet $H$, Crielaard W, Laheij A. A scoping review on bioaerosols in healthcare and the dental environment. PLoS One. 2017 May 22;12(5):e0178007. doi: 10.1371/journal.pone.0178007.

25. Houston Methodist Leading Medicine [Internet]. Texas [cited 2021 Jan 13] Available from: https://www.houstonmethodist.org/research/covid19/ aerosol/.

26. Liu L, Wei Q, Alvarez X, Wang H, Du Y, Zhu H, et al. Epithelial cells lining salivary gland ducts are early target cells of severe acute respiratory syndrome coronavirus infection in the upper respiratory tracts of rhesus macaques. J Virol. 2011 Apr;85(8):4025-30. doi: 10.1128/JVI.02292-10.

27. Chen D, Xu W, Lei Z, Huang Z, Liu J, Gao Z, et al. Recurrence of positive SARS CoV-2 RNA in COVID-19: A case report. Int J Infect Dis. 2020 Apr;93:297-9. doi: 10.1016/j.jiji.2020.03.003.

28. Sezgin GP, Şirino lu Çapan B. Assessment of dentists' awareness and knowledge levels on the Novel Coronavirus (COVID-19). Braz Oral Res. 2020:34:e112. doi: 10.1590/1807-3107bor-2020.vol34.0112.

29. Kamate SK, Sharma S, Thakar S, Srivastava D, Sengupta K, Hadi AJ, et al. Assessing knowledge, attitudes and practices of dental practitioners regarding the COVID-19 pandemic: a multinational study. Dent Med Probl. 2020 Jan-Mar;57(1):11-7. doi: 10.17219/dmp/119743.

30. Singh Gambhir R, Singh Dhaliwal J, Aggarwal A, Anand S, Anand V, Kaur Bhangu A. Covid-19: a survey on knowledge, awareness and hygiene practices among dental health professionals in an Indian scenario. Rocz Panstw Zakl Hig. 2020;71(2):223-9. doi: 10.32394/rpzh.2020.0115.
31. Kinariwala N, Samaranayake L, Perera I, Patel Z. Knowledge, awareness and perceptions of coronavirus disease 2019 (COVID-19) in a cohort of Indian dental professionals: a questionnaire-based study. Preprints. 2020;2020050442

32. Quadri FA, Jafer MA, Alqahtani AS, Mutahar SAB, Odabi NI, Daghriri AA, et al. Novel corona virus disease (COVID-19) awareness among the dental interns, dental auxiliaries and dental specialists in Saudi Arabia: a nationwide study.. J Infect Public Health. 2020 Jun;13(6):856-64. doi: 10.1016/j.jph.2020.05.010.

33. Barker J, Jones MV. The potential spread of infection caused by aerosol contamination of surfaces after flushing a domestic toilet. J Appl Microbiol. 2005;99(2):339-47. doi: 10.1111/j.1365-2672.2005.02610.x.

34. ASHRAE. ASHRAE position document on airbone infectious diseases. 2014. [Internet] Avaiable at: https://www.ashrae.org/file\%20library/about/ position\%20documents/airborne-infectious-diseases.pdf [Access: 03 Sep 2021]

35. Manocha A, Tak L. A study to assess the level of awareness among dental health professionals (DHPs) concerning current pandemic of Covid-19 in Jaipur City. IJIRMPS 2020;8:18-21.

36. Veena HR, Mahantesha S, Joseph AP,Patil SR, Patil SH. Dissemination of aerosol and splatter during ultrasonic scaling: a pilot study. J Infect Public Health. 2015 May-Jun;8(3):260-5. doi: 10.1016/j.jph.2014.11.004

37. Patil S, Moafa IH, Bhandi S, Jafer MA, Khan SS, Khan S, et al. Dental care and personal protective measures for dentists and non-dental health care workers. Dis Mon. 2020 Sep;66(9):101056. doi: 10.1016/j. disamonth.2020.101056

38. Madarati A, Abid S, Tamimi F, Ezzi A, Sammani A, Al Shaar MBA, et al. Dental-dam for infection control and patient safety during clinical endodontic treatment: Preferences of dental patients. Int J Environ Res Public Health. 2018 Sep 14;15(9):2012. doi: 10.3390/ijerph15092012.

\section{Merve Benli}

\section{(Corresponding address)}

University of Pittsburgh, School of Dental Medicine, Department of Oral Biology,

Pittsburgh, USA.

Email: benlimerve@hotmail.com

Date submitted: 2020 0ct 25

Accept submission: 2021 Feb 01 